Gamow-Teller response in the configuration space of a density-functional-theory-rooted no-core configuration-interaction model

Konieczka, M.

2018-03-07

Konieczka, M , Kortelainen , M \& Satula , W 2018 , ' Gamow-Teller response in the configuration space of a density-functional-theory-rooted no-core configuration-interaction model ' , Physical Review C , vol. 97 , no. 3 , 034310 . https://doi.org/10.1103/PhysRevC.97.034310

http://hdl.handle.net/10138/234772

https://doi.org/10.1103/PhysRevC.97.034310

cc_by

publishedVersion

Downloaded from Helda, University of Helsinki institutional repository.

This is an electronic reprint of the original article.

This reprint may differ from the original in pagination and typographic detail.

Please cite the original version. 


\title{
Gamow-Teller response in the configuration space of a density-functional-theory-rooted no-core configuration-interaction model
}

\author{
M. Konieczka, ${ }^{1}$ M. Kortelainen, ${ }^{2,3}$ and W. Satuła ${ }^{1,3}$ \\ ${ }^{1}$ Institute of Theoretical Physics, Faculty of Physics, University of Warsaw, ul. Pasteura 5, PL-02-093 Warsaw, Poland \\ ${ }^{2}$ Department of Physics, P.O. Box 35 (YFL), University of Jyvaskyla, FI-40014 Jyvaskyla, Finland \\ ${ }^{3}$ Helsinki Institute of Physics, P.O. Box 64, FI-00014 University of Helsinki, Finland
}

(Received 17 October 2017; published 7 March 2018)

\begin{abstract}
Background: The atomic nucleus is a unique laboratory in which to study fundamental aspects of the electroweak interaction. This includes a question concerning in medium renormalization of the axial-vector current, which still lacks satisfactory explanation. Study of spin-isospin or Gamow-Teller (GT) response may provide valuable information on both the quenching of the axial-vector coupling constant as well as on nuclear structure and nuclear astrophysics.

Purpose: We have performed a seminal calculation of the GT response by using the no-core configurationinteraction approach rooted in multireference density functional theory (DFT-NCCI). The model treats properly isospin and rotational symmetries and can be applied to calculate both the nuclear spectra and transition rates in atomic nuclei, irrespectively of their mass and particle-number parity.

Methods: The DFT-NCCI calculation proceeds as follows: First, one builds a configuration space by computing relevant, for a given physical problem, (multi)particle-(multi)hole Slater determinants. Next, one applies the isospin and angular-momentum projections and performs the isospin and $K$ mixing in order to construct a model space composed of linearly dependent states of good angular momentum. Eventually, one mixes the projected states by solving the Hill-Wheeler-Griffin equation.

Results: The method is applied to compute the GT strength distribution in selected $N \approx Z$ nuclei including the $p$-shell ${ }^{8} \mathrm{Li}$ and ${ }^{8} \mathrm{Be}$ nuclei and the $s d$-shell well-deformed nucleus ${ }^{24} \mathrm{Mg}$. In order to demonstrate a flexibility of the approach we present also a calculation of the superallowed GT $\beta$ decay in doubly-magic spherical ${ }^{100} \mathrm{Sn}$ and the low-spin spectrum in ${ }^{100} \mathrm{In}$.

Conclusions: It is demonstrated that the DFT-NCCI model is capable of capturing the GT response satisfactorily well by using a relatively small configuration space, exhausting simultaneously the GT sum rule. The model, due to its flexibility and broad range of applicability, may either serve as a complement or even as an alternative to other theoretical approaches, including the conventional nuclear shell model.
\end{abstract}

DOI: 10.1103/PhysRevC.97.034310

\section{INTRODUCTION}

Single reference density functional theory (SR-DFT) has proved to be extremely successful in accounting for bulk nuclear properties such as masses, radii, or quadrupole moments over the entire nuclear chart; see [1,2] and references quoted therein. The success of SR-DFT or, alternatively, selfconsistent mean-field theory has its roots in the spontaneous symmetry breaking, which allows one to incorporate correlations into a single Slater determinant. The deformed wave function does not allow, however, for quantum-mechanically rigorous treatment of either the nuclear spectra or the nuclear decay rates. So far, this domain has been traditionally reserved for the nuclear shell model (NSM), a configuration-interaction (CI) approach involving strict laboratory-frame treatment of symmetries; see [3] for a review.

An expansion of applicability of the mean-field or single reference energy density functional (SR-EDF) based methods is ultimately related to symmetry restoration. Recently, strenuous effort was devoted to a development of symmetry-projected multireference DFT (MR-DFT) and to extend it towards the no-core configuration-interaction (NCCI) approach. Bally and coworkers proposed a DFT-NCCI framework involving a Skyrme superfluid functional and applied it successfully to compute spectra and electromagnetic transition rates in ${ }^{25} \mathrm{Mg}$ [4]. Our group has developed a variant involving an unpaired Skyrme functional and a unique combination of angular-momentum and isospin projections and applied to calculate the spectra and $\beta$-decay rates in $N \approx Z$ nuclei from $p$-shell to medium mass nuclei around ${ }^{62} \mathrm{Zn}$ [5-8]. Recently, the DFT-NCCI method was applied to calculate spectra in neutron-rich ${ }^{44} \mathrm{~S}$ and ${ }^{64} \mathrm{Cr}$ nuclei with the Gogny force $[9,10]$, within the relativistic framework [11] in ${ }^{54} \mathrm{Cr}$, or within the pairing-plus-quadrupole model in the magnesium chain [12].

The results obtained so far have been very promising. In particular, they indicate that a relatively limited number of configurations is needed to obtain an accurate description of low-energy, low-spin physics in complex nuclei. However, further tests of these methods are still required.

The DFT-NCCI method allows one to address many important physics questions in a way that is complementary to the conventional NSM. The flagship example concerns physical origin of the quenching effect of the weak axial 
coupling constant [ for free-neutron decay $g_{\mathrm{A}}=-1.2701(25)$ ], a subject of intense discussion since the first Gamow-Teller (GT) $\beta$-decay calculations were performed. The DFT-NCCI calculations in $T=1 / 2$ mirror nuclei [8] somewhat contradict the statement that the quenching has its roots in a model space, and therefore support the two-body current based explanation, put forward in Refs. [13,14]; see also [15-18].

The goal of this work is to compute spin-isospin response by using, for the first time, the DFT-NCCI approach. The spin-isospin, or GT, response provides valuable information on both the electroweak $\beta$ decay and nuclear structure. Since DFT-NCCI originates from the very intuitive and powerful concept of spontaneous symmetry breaking, it gives a unique opportunity to discuss complex patterns that emerge in the response function in terms of simple deformed single-particle Nilsson levels which are the primary building blocks of the formalism. In this sense the DFT-NCCI can be considered again as a complementary method with respect to the NSM $[3,19,20]$, coupled cluster method [14], or quasiparticle random phase approximation (QRPA) [21-26] which was, until now, the only possible mean-field-based alternative to the NSM concerning global studies of GT strength distribution. Last but not least, $\beta$ decay in $p f$-shell nuclei is studied in the variation-after-projection excited VAMPIR (variation after mean-field projection in realistic model spaces) approach with $G$-matrix-driven realistic effective interaction [27]. Although the method is based on a mean-field concept, its model space and treatment of correlations are entirely different from the DFT-NCCI model.

This paper is organized as follows. In Sec. II we discuss the foundations of the DFT-NCCI model, paying special attention to the concept of configuration and model spaces. In Sec. III we present the results for the structure and GT strength distribution in $A=8$ nuclei. In Sec. IV we discusses the spin-isospin response in the $s d$-midshell nucleus ${ }^{24} \mathrm{Mg}$. Eventually, in Sec. V, we focus on the ${ }^{100} \mathrm{Sn} \rightarrow{ }^{100}$ In superallowed GT $\beta$ decay and the low-spin spectrum of ${ }^{100} \mathrm{In}$. A summary and conclusions are presented in Sec. VI. All calculations presented in this work were done using developing version of the HFODD solver [28,29] equipped with the NCCI module.

\section{THE DFT-ROOTED NO-CORE CONFIGURATION-INTERACTION MODEL}

The DFT-NCCI models are post Hartree-Fock(-Bogliubov) approaches which mix nonorthogonal many-body states projected from symmetry breaking mean-field solutions. Their sole ingredients are therefore independent-particle (quasi)particle-(quasi)hole configurations and projection techniques that are used to restore spontaneously broken symmetries. In practical applications, the projections are handled by using the generalized Wick's theorem (GWT) which leads from a SR to multireference (MR) formulation of the DFT (MR-DFT).

The GWT allows one to handle theory numerically; however, it leads to singular kernels once modern densitydependent Skyrme or Gogny forces are used for the beyondmean-field part of the calculation. The intensive work to overcome this problem of projection-induced singularities is currently under way. The attempts to regularize the kernels $[30,31]$ have not provided a satisfactory solution so far. Hence, at present, the theory can be safely carried on only for true interaction such as $\mathrm{SV}_{\mathrm{T}}$ [32], used in the present work, or the SLyM0 [33], which both are density-independent Skyrme pseudopotentials. In addition to these, the recently developed regularized finite-range pseudopotential [34] aims also for beyond-mean-field calculations. It is worth mentioning that these pseudopotentials are characterized by anomalously low effective mass, which affects the single-particle (s.p.) level density and, in turn, influences spectroscopic properties of the calculated nuclei.

The MR-DFT approach developed by our group is unique in the sense that it restores angular momentum and treats rigorously the isospin symmetry, i.e., retains only physical sources of its breaking. It provides wave functions which are isospin $(T)$ and $K$ (projection of angular momentum onto intrinsic $z$ axis) mixed as

$$
\left|\varphi ; I M ; T_{z}\right\rangle^{(i)}=\frac{1}{\sqrt{\mathcal{N}_{\varphi ; I M ; T_{z}}^{(i)}}} \sum_{\substack{K, T \geqslant\left|T_{z}\right|}} a_{K T}^{(i)} \hat{P}_{T_{z} T_{z}}^{T} \hat{P}_{M K}^{I}|\varphi\rangle,
$$

where

$$
\begin{gathered}
\hat{P}_{T_{z} T_{z}}^{T}=\frac{2 T+1}{2} \int_{0}^{\pi} d \beta_{T} \sin \beta_{T} d_{T_{z} T_{z}}^{T}\left(\beta_{T}\right) e^{-i \beta_{T} \hat{T}_{y}}, \\
\hat{P}_{M K}^{I}=\frac{2 I+1}{8 \pi^{2}} \int d \Omega D_{M K}^{I *}(\Omega) e^{-i \gamma \hat{J}_{z}} e^{-i \beta \hat{J}_{y}} e^{-i \alpha \hat{J}_{z}}
\end{gathered}
$$

are the isospin and angular-momentum projection operators, respectively. Above, the index $i$ enumerates different states of a given spin $I, \mathcal{N}_{\varphi ; I M ; T_{z}}^{(i)}$ is a normalization constant, while $d_{T_{z} T_{z}}^{T}\left(\beta_{T}\right)$ and $D_{M K}^{I}(\Omega)$ stand for the Wigner functions. The integration over the Euler angles in space $\Omega=(\alpha, \beta, \gamma)$ and isospace $\beta_{T}$ is performed using Gauss quadratures with $n_{\alpha}=$ $n_{\beta}=n_{\gamma}=20$ knots in space and $n_{\beta_{T}}=10$ knots in isospace; see [28] for further details. This setup assures very precise integration over the Euler angles for all the low-spin states considered in this work, with the exception of the $I=8^{+}$ state in ${ }^{100} \mathrm{In}$, which might be somewhat uncertain as discussed below.

The Slater determinant, $\varphi$, is calculated self-consistently by using the Hartree-Fock (HF) method in the Cartesian harmonic oscillator $(\mathrm{HO})$ basis with the $\mathrm{SV}_{\mathrm{T}}$ Skyrme and Coulomb forces; see [28,29] and references quoted therein. In the calculations presented below we use a basis composed of 10 spherical HO shells for the $p$ - and $s d$-shell nuclei, whereas, for the $p f$-shell and heavier nuclei, we use 12 spherical HO shells. The exchange term of the Coulomb interaction is treated exactly.

Although the effect of isospin mixing on the calculated quantities is expected to be rather small, the use of isospin projection is indispensable in computing $\beta$-decay matrix elements. Indeed, these matrix elements connect states built upon orthogonal (different nuclei) Slater determinants and are therefore not amenable for direct computation based on the GWT. The restoration of isospin symmetry, by rotating one of these Slater determinants in isospace, allows one to detour 
around this obstacle in a simple, physically well motivated, and elegant way.

The MR-DFT wave functions (1) can be successfully used to compute, for example, $\beta$-decay transition rates between the ground states as shown in Refs. $[8,35]$. In order to account for $\beta$-decay strength distribution, the MR-DFT concept needs to be extended by including the states (1) projected from many Slater determinants $\varphi_{j}$ corresponding to different (multi)particle-(multi)hole excitations. The projected states, which are generally nonorthogonal to each other, are mixed by solving the Hill-Wheeler-Griffin equation with, typically, the same Hamiltonian that was used to generate them at the HF stage [36]. In effect, one obtains a set of linearly independent DFT-NCCI eigenstates of the form

$$
\left|\psi_{\mathrm{NCCI}}^{k ; I M ; T_{z}}\right\rangle=\frac{1}{\sqrt{\mathcal{N}_{I M ; T_{z}}^{(k)}}} \sum_{i j} c_{i j}^{(k)}\left|\varphi_{j} ; I M ; T_{z}\right\rangle^{(i)}
$$

together with the corresponding energy spectrum. More details concerning our method can be found in Ref. [7].

Contrary to the standard NSM, the model space of our DFT-NCCI approach is not fixed. It is built step by step, by adding physically relevant low-lying particle-hole (p-h) mean-field configurations which correspond to self-consistent HF solutions conserving parity and signature symmetries. The basic idea is to explore all relevant single-particle Nilsson levels. Hence, in even-even nuclei, we include in the first place the ground-state configuration and low-lying aligned $(|h\rangle \otimes$ $|\tilde{p}\rangle$ or $|\tilde{h}\rangle \otimes|p\rangle)$ and anti-aligned $(|h\rangle \otimes|p\rangle$ or $|\tilde{h}\rangle \otimes|\tilde{p}\rangle)$ 1p-1h configurations, where $|p\rangle$ and $|\tilde{p}\rangle(|h\rangle$ and $|\tilde{h}\rangle)$ label single-particle (single-hole) states of opposite signature. In an odd- $A$ nuclei we explore first configurations built by exciting the unpaired nucleon within a fixed signature block. In the second step we test stability of the predictions with respect to low-lying broken-pair configurations. Similar strategy is used in odd-odd nuclei. In this case, however, one has to consider both aligned and anti-aligned configurations.

In most of the applications, isospin symmetry restoration allows one to reduce the configuration space in $N=Z$ nuclei by a factor of 2 due to similarity between the neutron and proton $1 \mathrm{p}-1 \mathrm{~h}$ excitations. The effect is illustrated in Fig. 1 for a representative example of ${ }^{24} \mathrm{Mg}$. In the present calculation, the SR ground state (g.s.) and the lowest proton $(\pi \mathrm{p}-\pi \mathrm{h})$ and neutron ( $\nu \mathrm{p}-\nu \mathrm{h}) 1 \mathrm{p}-1 \mathrm{~h}$ HF configurations were taken into account. Energies of excited states differ by $80 \mathrm{keV}$ as shown in the left column of Fig. 1. By applying the angular-momentum and isospin projections with $I=4^{+}$, one obtains the corresponding, symmetry restored $I=4_{1}^{+}$from the ground state and four almost doubly degenerated excited $I=4^{+}$states, as shown in the second column of Fig. 1. The third and fourth columns show the DFT-NCCI results. The third column depicts configuration mixing calculation involving two HF configurations: the g.s. and the lowest neutron $1 \mathrm{p}-1 \mathrm{~h}$ excitation. The fourth column shows the results of three-configuration mixing, including, in addition to the previous case, the lowest proton $1 \mathrm{p}-1 \mathrm{~h}$ excitation. Addition of the proton $1 \mathrm{p}-1 \mathrm{~h}$ configuration almost does not influence either the spectrum or the GT matrix elements for $\left|{ }^{24} \mathrm{Al} ; 4_{1}^{+}\right\rangle \rightarrow\left|{ }^{24} \mathrm{Mg} ; 4_{i}^{+}\right\rangle$decay.

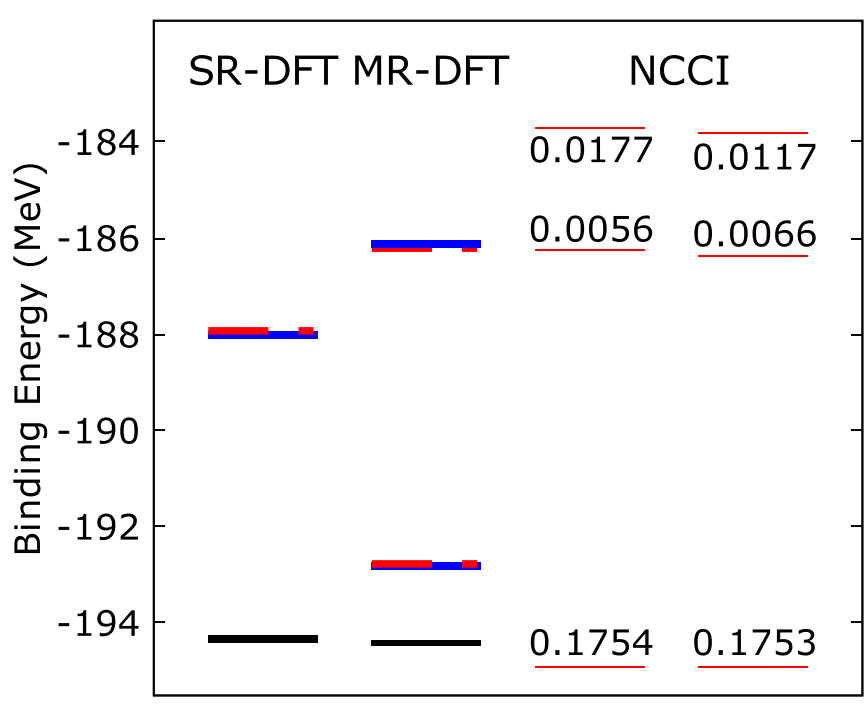

FIG. 1. The leftmost column shows the HF energies of the g.s. and the lowest $\nu \mathrm{p}-\nu \mathrm{h}$ (red, dashed line) and $\pi \mathrm{p}-\pi \mathrm{h}$ (blue, solid line) excitations in ${ }^{24} \mathrm{Mg}$. The second column illustrates $4^{+}$states projected from these SR configurations without configuration mixing. The last two columns depict the DFT-NCCI results involving different configurations. The left (right) part shows the CI results involving the g.s. and $\nu \mathrm{p}-\nu \mathrm{h}(\pi \mathrm{p}-\pi \mathrm{h})$ configurations, respectively. Numbers in the last two columns indicate the calculated GT matrix elements for $\left.{ }^{24} \mathrm{Al} ; 4_{1}^{+}\right\rangle \rightarrow\left|{ }^{24} \mathrm{Mg} ; 4_{i}^{+}\right\rangle$decay.

\section{LOW-ENERGY SPECTRA AND GAMOW-TELLER $\beta$ DECAY FOR $A=8$ NUCLEI}

In this section we will investigate the structure and $\beta$ decay properties of very light nuclei ${ }^{8} \mathrm{Be},{ }^{8} \mathrm{Li}$, and ${ }^{8} \mathrm{He}$ by using the DFT-NCCI framework. The $p$-shell nuclei offer an excellent playground to test, in particular, a configurationspace dependence of our scheme. One should bear in mind, however, that light nuclei are weakly bound. Hence, they may exhibit a variety of phenomena which either emerge or strongly depend on the coupling to continuum $[37,38]$, which is beyond our approach. These effects include clustering, appearance of low-lying broad resonances or particle-decay channels that may compete with $\beta$ decay and, in turn, significantly influence $\beta$-decay strength distribution.

We shall focus on GT strength distributions of ${ }^{8} \mathrm{He}, 0_{\text {g.s. }}^{+}$ and ${ }^{8} \mathrm{Li}, 2_{\text {g.s. }}^{+} \beta$ decays, paying special attention to the physical interpretation of particular peaks. For the first time these peaks can be interpreted in terms of deformed Nilsson states and deformed Nilsson configurations used in the mixing. We shall also investigate the saturation of GT sum rules for the lowest $1^{+}, 2^{+}$, and $3^{+}$states in ${ }^{8} \mathrm{Li}$ in order to verify the completeness of the model space.

\section{A. Configuration space in $A=8$ nuclei}

Let us start the discussion by recalling the strategy of building configuration space. As already discussed in Sect. II, we start by calculating self-consistently the HF g.s. configuration. The s.p. Nilsson levels of both signatures (the signature symmetry is superimposed on our HF solutions) in the g.s. 

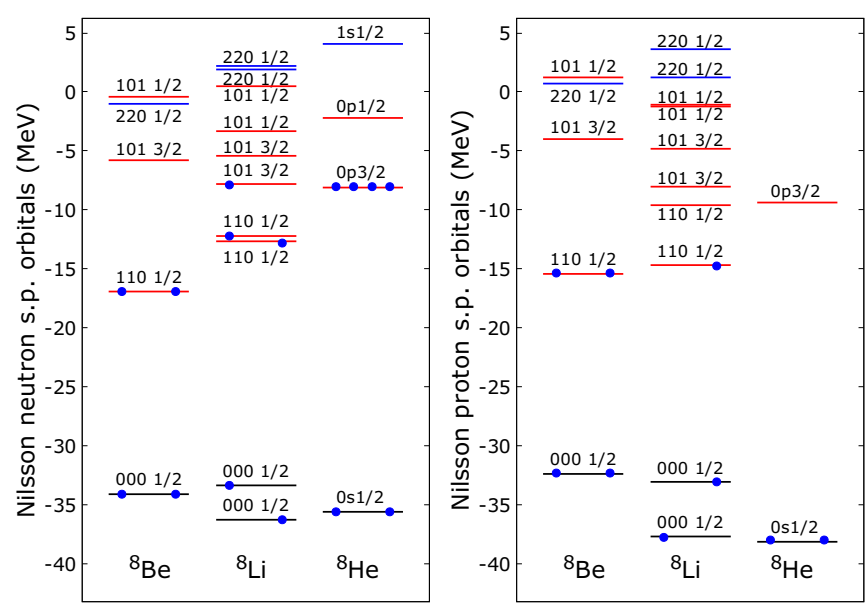

FIG. 2. Nilsson neutron (left) and proton (right) mean-field single-particle orbitals for the SR ground state of ${ }^{8} \mathrm{Be},{ }^{8} \mathrm{Li}$, and ${ }^{8} \mathrm{He}$. The orbitals are labeled with approximate Nilsson quantum numbers. Dots indicate occupied levels, and colors differentiate orbitals between parity blocks.

are used next as a guide to construct excited configurations. First we include all relevant $1 \mathrm{p}-1 \mathrm{~h}$ configurations. If needed, we extend the configuration space by adding low-lying $2 \mathrm{p}-2 \mathrm{~h}$ configurations, etc.

The neutron and proton s.p. Nilsson levels calculated for the ground states of ${ }^{8} \mathrm{Be},{ }^{8} \mathrm{Li}$, and ${ }^{8} \mathrm{He}$ are shown in Fig. 2 . Note that the g.s. configurations of ${ }^{8} \mathrm{Be}$ and ${ }^{8} \mathrm{Li}$ are well deformed while the g.s. of ${ }^{8} \mathrm{He}$ is spherical. The split of s.p. level energies in ${ }^{8} \mathrm{Li}$ is due to breaking of the time-reversal symmetry. In the case of $N=Z$ nucleus ${ }^{8} \mathrm{Be}$, we built the space by taking into account the g.s. configuration. Next, we attempt to compute all four possible (aligned and anti-aligned) neutron $1 \mathrm{p}-1 \mathrm{~h}$ excitations among the available $\left|N=1 n_{z} \Lambda \Omega \pm\right\rangle$ Nilsson states, where \pm refers to the signature quantum number $r= \pm i$. It appears, however, that one of them, the anti-aligned excitation to the first Nilsson s.p. orbital $|1013 / 2\rangle$, does not converge. Eventually, in an attempt to cover the missing correlations from the s.p orbital $|1013 / 2\rangle$, the configuration space consisting of the g.s. and three $\nu \mathrm{p}-\nu \mathrm{h}$ is extended by adding three lowest $2 \mathrm{p}-2 \mathrm{~h}$ excitations.

In the semimagic nucleus ${ }^{8} \mathrm{He}$ we include in the model space the g.s. and four $1 \mathrm{p}-1 \mathrm{~h}$ neutron excitations.

In odd-odd ${ }^{8} \mathrm{Li}$, we compute first the aligned and antialigned g.s. configurations. Next, keeping the two neutrons paired in the lowest available signature reversed Nilsson states, we calculate several possible excited $|\nu\rangle \otimes|\pi\rangle$ configurations by distributing the unpaired proton and unpaired neutron over the available s.p. states. Eventually, we break the neutron pair and attempt to compute fully unpaired configurations. These configurations are highly excited and difficult to converge. We were able to converge two such low- $K$ axial configurations. As will be shown below, in Sec. III, they do not influence the low-energy part of the spectrum but have quite significant impact on the GT resonance.

All configurations included in the configuration spaces of ${ }^{8} \mathrm{Be},{ }^{8} \mathrm{Li}$, and ${ }^{8} \mathrm{He}$ are listed in Table I. The configurations are labeled by means of the Nilsson and signature quantum
TABLE I. Mean-field self-consistent configurations in ${ }^{8} \mathrm{He},{ }^{8} \mathrm{Be}$, and ${ }^{8} \mathrm{Li}$. Configurations are ordered according to their excitation energies (index $i$ ) and labeled by the asymptotic Nilsson quantum numbers and the signature of unpaired valence particles and holes. The last four columns list their properties, including HF energy in $\mathrm{MeV}$, quadrupole deformation parameters $\beta_{2}$ and $\gamma$, and the total alignment $\langle j\rangle$ and its orientation in the intrinsic frame, respectively.

\begin{tabular}{|c|c|c|c|c|c|}
\hline$i$ & $\left|{ }^{8} \mathrm{He} ; \varphi_{i}\right\rangle$ & $E_{\mathrm{HF}}$ & $\beta_{2}$ & $\gamma$ & $\langle j\rangle$ \\
\hline 1 & $v p_{3 / 2} \otimes \pi s_{1 / 2}$ & -37.26 & 0 & $0^{\circ}$ & 0 \\
\hline 2 & $|\nu 1013 / 2-\rangle^{-1} \otimes|v 1011 / 2+\rangle^{1}$ & -32.47 & 0.14 & $0^{\circ}$ & $2_{Z}$ \\
\hline 3 & $|v 1013 / 2+\rangle^{-1} \otimes|v 1011 / 2-\rangle^{1}$ & -30.81 & 0.03 & $60^{\circ}$ & $1_{Y}$ \\
\hline 4 & $|v 1101 / 2+\rangle^{-1} \otimes|v 1011 / 2+\rangle^{1}$ & -30.04 & 0.03 & $60^{\circ}$ & $0_{Y}$ \\
\hline 5 & $|\nu 1101 / 2+\rangle^{-1} \otimes|v 1011 / 2-\rangle^{1}$ & -29.13 & 0.02 & $0^{\circ}$ & $1_{Z}$ \\
\hline$i$ & $\left|{ }^{8} \mathrm{Be} ; \varphi_{i}\right\rangle$ & $E_{\mathrm{HF}}$ & $\beta_{2}$ & $\gamma$ & $\langle j\rangle$ \\
\hline 1 & $|\nu 1101 / 2\rangle^{2} \otimes|\pi 1101 / 2\rangle^{2}$ & -48.66 & 0.68 & $0^{\circ}$ & $\overline{0_{Z}}$ \\
\hline 2 & $|\nu 1101 / 2-\rangle^{-1} \otimes|v 1013 / 2+\rangle^{1}$ & -38.87 & 0.40 & $0^{\circ}$ & $1_{Z}$ \\
\hline 3 & $|\nu 1101 / 2-\rangle^{-1} \otimes|v 1011 / 2+\rangle^{1}$ & -34.08 & 0.39 & $0^{\circ}$ & $1_{Y}$ \\
\hline 4 & $|v 1101 / 2+\rangle^{-1} \otimes|v 1011 / 2+\rangle^{1}$ & -31.63 & 0.27 & $3^{\circ}$ & $0.7_{Z}$ \\
\hline 5 & $\begin{array}{l}|v 1101 / 2+\rangle^{-1} \otimes|v 1013 / 2+\rangle^{1} \\
|\pi 1101 / 2+\rangle^{-1} \otimes|\pi 1013 / 2+\rangle^{1}\end{array}$ & -36.81 & 0.20 & $60^{\circ}$ & $0_{Z}$ \\
\hline 6 & $|v 1101 / 2\rangle^{-2} \otimes|v 1013 / 2\rangle^{2}$ & -35.74 & 0.11 & $5^{\circ}$ & $0_{Z}$ \\
\hline 7 & $\begin{array}{l}|\nu 1101 / 2+\rangle^{-1} \otimes|v 1013 / 2+\rangle^{1} \\
|\pi 1101 / 2+\rangle^{-1} \otimes|\pi 1013 / 2+\rangle^{1}\end{array}$ & -34.28 & 0.12 & $0^{\circ}$ & $2 z$ \\
\hline$i$ & $\left|{ }^{8} \mathrm{Li} ; \varphi_{i}\right\rangle$ & $E_{\mathrm{HF}}$ & $\beta_{2}$ & $\gamma$ & $\langle j\rangle$ \\
\hline$\overline{1}$ & $|v 1013 / 2+\rangle \otimes|\pi 1101 / 2+\rangle$ & -39.08 & 0.38 & $0^{\circ}$ & $\overline{1_{Z}}$ \\
\hline 2 & $|v 1013 / 2+\rangle \otimes|\pi 1101 / 2-\rangle$ & -39.03 & 0.36 & $0^{\circ}$ & $2 z_{Z}$ \\
\hline 3 & $|v 1011 / 2+\rangle \otimes|\pi 1101 / 2+\rangle$ & -34.04 & 0.36 & $0^{\circ}$ & $1_{Z}$ \\
\hline 4 & $|\nu 1011 / 2-\rangle \otimes|\pi 1101 / 2+\rangle$ & -33.44 & 0.35 & $0^{\circ}$ & $0_{Z}$ \\
\hline 5 & $|\nu 1101 / 2+\rangle \otimes|\pi 1101 / 2-\rangle$ & -36.51 & 0.07 & $60^{\circ}$ & $0_{Z}$ \\
\hline 6 & $|\nu 1013 / 2+\rangle \otimes|\pi 1013 / 2+\rangle$ & -35.68 & 0.03 & $0^{\circ}$ & $0_{Y}$ \\
\hline 7 & $|\nu 1013 / 2+\rangle \otimes|\pi 1011 / 2-\rangle$ & -32.34 & 0.12 & $0^{\circ}$ & $2_{Z}$ \\
\hline 8 & $|v 1011 / 2+\rangle \otimes|\pi 1101 / 2+\rangle$ & -31.19 & 0.06 & $60^{\circ}$ & $1_{Z}$ \\
\hline 9 & $\begin{array}{c}|\nu 1013 / 2+\rangle \otimes|\nu 1101 / 2+\rangle \\
\otimes|v 1011 / 2-\rangle \otimes|\pi 1013 / 2-\rangle\end{array}$ & -29.25 & 0.04 & $60^{\circ}$ & $0_{Y}$ \\
\hline 10 & $\begin{array}{c}|v 1013 / 2+\rangle \otimes|v 1101 / 2+\rangle \\
\otimes|v 1011 / 2+\rangle \otimes|\pi 1013 / 2-\rangle\end{array}$ & -29.06 & 0.07 & $60^{\circ}$ & $1_{Y}$ \\
\hline
\end{tabular}

numbers, $\left|N n_{z} \Lambda \Omega \pm\right\rangle$, pertaining to the unpaired valence particles. The table also includes quadrupole deformation parameters $\beta_{2}$ and $\gamma$ for each configuration. A value of $\gamma \neq 0^{\circ}$ or $\gamma \neq 60^{\circ}$ indicates a triaxial configuration.

We use the Nilsson quantum numbers to label not only deformed but also near-spherical configurations. This is partly justified since some of these configurations, in particular those in ${ }^{8} \mathrm{Li}$, exhibit very peculiar isovector shape effects. For example, the near-spherical configuration 6 in ${ }^{8} \mathrm{Li}$ is a superposition of prolate (oblate) density distribution of neutrons (protons), respectively, while the configurations 5 and 8 are superpositions of oblate (prolate) density distributions of neutrons (protons), respectively. The near-spherical configurations 7, 9, and 10, on the other hand, are built of near-spherical density distribution of protons 7 (neutrons 9 and 10) and deformed 


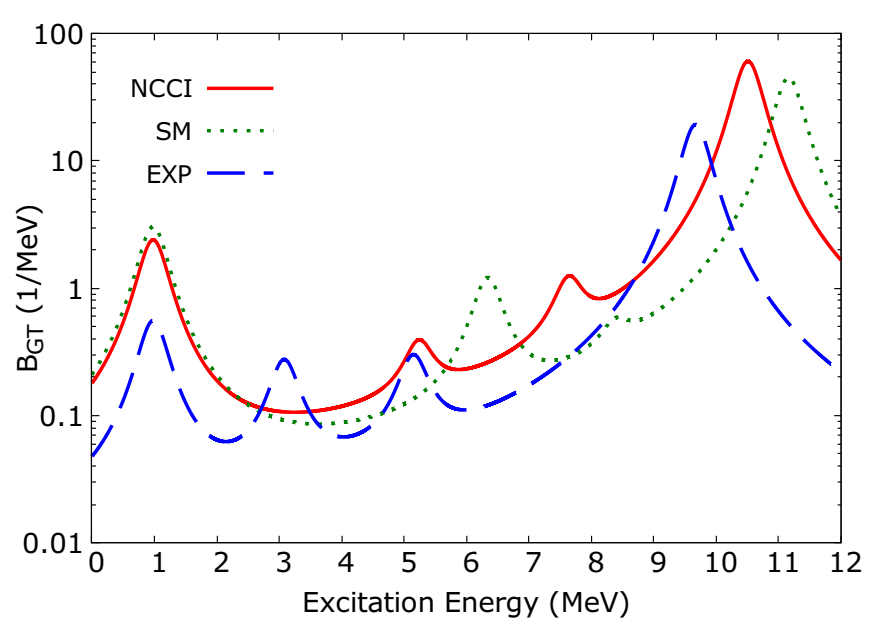

FIG. 3. The GT strength distribution in $1^{+}$states of ${ }^{8} \mathrm{Li}$ in logarithmic scale smoothed with a Lorentzian function with halfwidth of $\Gamma=0.5 \mathrm{MeV}$. The dashed curve represents experimental data obtained by means of the $R$-matrix theory in Ref. [39,40]. The dotted line marks the shell-model input to the $R$ matrix [39] calculated using the $p$-shell residual interaction proposed by Kumar in Ref. [41]. The continuous line labels the DFT-NCCI calculations. See text for discussion.

density distribution of neutrons (protons), respectively. Note, that these isovector shape effects may lead to different $\Omega$ ordering of the neutron and proton s.p. levels.

\section{B. GT strength distribution for ${ }^{8} \mathrm{He}$ to ${ }^{8} \mathrm{Li}$ decay}

$\beta$ decay from the $0^{+}$g.s. of ${ }^{8} \mathrm{He}$ populates four $1^{+}$states in ${ }^{8} \mathrm{Li}$ within the experimental $Q_{\beta}$ energy window. Except for the lowest $1^{+}$state, the remaining $1^{+}$states may decay through different particle emission channels. This makes, therefore, both the energy and $B_{\mathrm{GT}}$ of decaying states extremely difficult to determine experimentally. In fact, the so-called experimental determination of $\beta$-decay properties of ${ }^{8} \mathrm{He}$ is based on the multiparameter $R$-matrix formalism. The initial values of the $R$-matrix parameters are taken from shell-model calculations. These parameters are varied next to best fit the available data on half-life, branching ratios, and energy spectra of $\beta$-delayed particles $[39,42,43]$. The inclusion of the particle emission channels reduces the experimental $B_{\mathrm{GT}}$ to the resonant $1^{+}$ states in ${ }^{8} \mathrm{Li}$ and shifts their energies (centroids) compared to the initial shell-model values, with the largest impact on the GT resonance-the fourth $1^{+}$state-which can decay through both neutron and triton emission, as shown in Fig. 3.

In Fig. 3 we present GT strength function for the decay of $0^{+}$g.s. of ${ }^{8} \mathrm{He}$, smoothed with the Lorentzian distribution with a half-width of $\Gamma=0.5 \mathrm{MeV}$. The peaks in the distribution reflect excitation energies of the GT-populated $1^{+}$states in ${ }^{8} \mathrm{Li}$. Distribution is normalized with respect to the first $1^{+}$state which is bound.

The DFT-NCCI model predicts a $2^{+}$g.s. in ${ }^{8} \mathrm{Li}$ at the energy of $-41.9 \mathrm{MeV}$, which is only $\sim 0.6 \mathrm{MeV}$ below the experimental value. The resonant peak in the DFT-NCCI spectrum is shifted by $\sim 1 \mathrm{MeV}$ towards higher energies as compared to experimental data, whereas the second and third

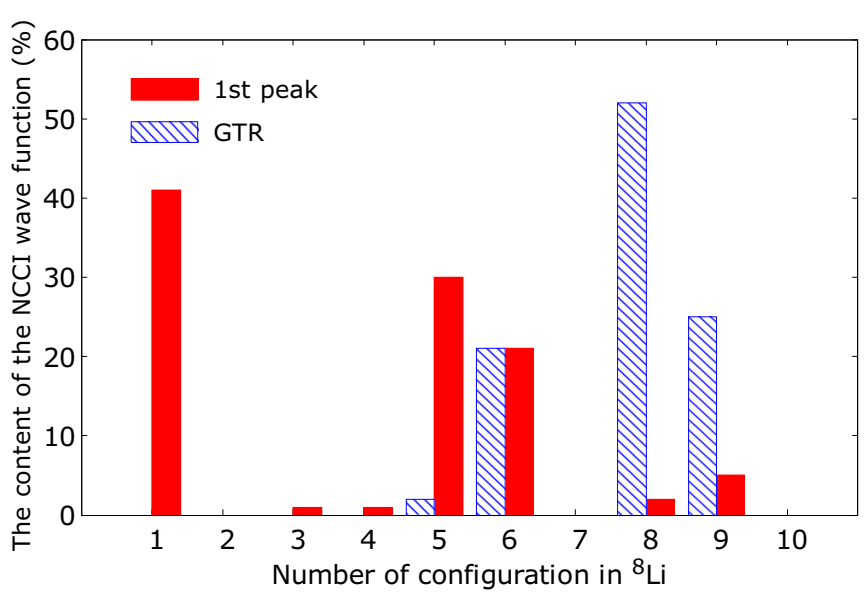

FIG. 4. Decomposition of the wave functions of the first and fourth (GT resonance) $1^{+}$states in ${ }^{8} \mathrm{Li}$ in terms of the $\mathrm{HF}$ configurations, $\varphi_{n}$, included in the space. An abscissa numbers the configurations in accordance with Table I.

peaks are roughly $2 \mathrm{MeV}$ higher than the experiment. The height of the peaks is overestimated, in particular, for the GT resonance. Naturally, such a big difference cannot be explained solely by the quenching factor, which is a fortiori expected to be close to unity in light nuclei. The discrepancy is mostly due to lack of the coupling to particle-emission channels in the DFT-NCCI. In this respect, our results should be considered as an input to the $R$ matrix and compared directly to the shell-model input to the $R$ matrix. Such a comparison shows (see Fig. 3) that the results for the GT strength distribution are very similar. This is beneficial for us since our calculations are free from any adjustable parameter, at variance with the shell-model results of Ref. [41]. Part of the discrepancy may be also due to the three-nucleon forces which, in the $a b$ initio NCCI calculation, may become prominent, as was found in the $\beta$ decay of ${ }^{14} \mathrm{C}$ [44].

The DFT-NCCI approach allows for rather unique analysis of the GT strength distribution in terms of HF configurations, which are the primary building blocks of the model. This is particularly useful in deformed nuclei where HF configurations, corresponding to certain $\mathrm{p}$-h excitations, can be conveniently and rather intuitively labeled by Nilsson quantum numbers. The content of the $n$th HF configuration in the $k$ th DFT-NCCI state of a given $I$ and $T_{z}$ [see Eq. (4)] that corresponds to the $k$ th peak in the spectrum is given by the following formula:

$$
\begin{aligned}
P\left(\varphi_{n}\right) & \left.\left.\equiv \sum_{i}\right|^{(i)}\left\langle\varphi_{n} ; I M ; T_{z} \mid \psi_{\mathrm{NCCI}}^{k ; I M ; T_{z}}\right\rangle\right|^{2} \\
& =\frac{1}{\mathcal{N}_{I M ; T_{z}}^{(k)}} \sum_{i}\left|\sum_{j l} c_{j l}^{(k)}{ }^{(i)}\left\langle\varphi_{n} ; I M ; T_{z} \mid \varphi_{j} ; I M ; T_{z}\right\rangle^{(l)}\right|^{2} .
\end{aligned}
$$

Figure 4 shows a decomposition of the wave functions of the first and fourth $1^{+}$states in ${ }^{8} \mathrm{Li}$ in terms of the included HF configurations. These HF configurations are the same as those listed in Table I. As shown in the figure, the first peak is a mixture of the very well deformed aligned ground 


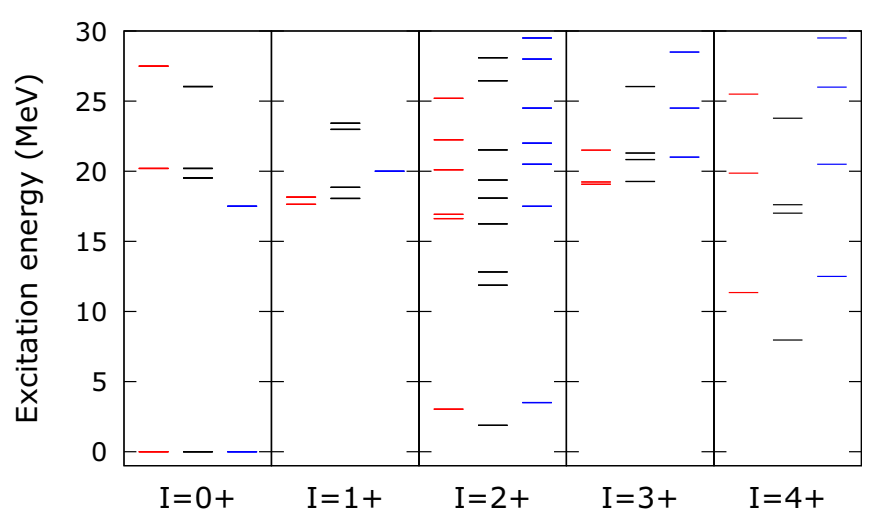

FIG. 5. Low-spin states in ${ }^{8}$ Be below $30 \mathrm{MeV}$. The panels show, counting from the left, the groups of levels having spins $I^{\pi}=0^{+}$, $1^{+}, 2^{+}, 3^{+}$, and $4^{+}$, respectively. Each panel shows experimental (left), DFT-NCCI (center), and ab initio NCCI (right) spectra, each normalized with respect to its g.s. energy.

state, $\left|{ }^{8} \mathrm{Li} ; \varphi_{1}\right\rangle$, with two very weakly deformed proton excitations, $i=5$ and 6 . The lowest proton-excited configuration corresponds to oblate shape. The second is a proton-excited configuration corresponding to prolate shape.

The resonance is centered around a weakly deformed oblate configuration, $\left|{ }^{8} \mathrm{Li} ; \varphi_{8}\right\rangle$, corresponding to $1 \mathrm{p}$ - $1 \mathrm{~h}$ aligned excitation from the $|v 1013 / 2+\rangle$ Nilsson level to its spin-orbit partner $|v 1011 / 2-\rangle$, with drastic shape change of neutron density. An admixture of broken-neutron-pair configuration 9 to the resonance is of the order of $25 \%$. And finally, $20 \%$ of a resonant peak comes from the lowest proton excitation to the $|\pi 1013 / 2+\rangle$ Nilsson level.

\section{GT strength distribution for ${ }^{8} \mathrm{Li}$ to ${ }^{8} \mathrm{Be}$ decay}

The ${ }^{8} \mathrm{Be}$ nucleus is a cluster composed of two $\alpha$ particles. Its molecular structure is characterized by very elongated distribution of nuclear matter, which is well accounted for by our mean-field calculation, which predicts a sudden increase of deformation in ${ }^{8} \mathrm{Be}$ to $\beta_{2}=0.68$ as compared to its neighbors. It appears, however, that neither the HF nor the DFT-NCCI can account for all correlations associated with the clustering. The g.s. energy calculated using the DFT-NCCI equals -52.8 $\mathrm{MeV}$, underestimating the experimental value by $3.7 \mathrm{MeV}$. This should be compared to the g.s. energy of ${ }^{8} \mathrm{Li}$, which was overestimated only by $0.6 \mathrm{MeV}$.

The low-spin positive-parity levels in ${ }^{8} \mathrm{Be}$ are shown in Fig. 5. Apart of experimental data and the results of DFT-NCCI, the figure includes also, for the sake of comparison, the results of $a b$ initio NCCI calculations of Ref. [45] with the JISP16 interaction. This calculation predicts the g.s. at $-57.5 \mathrm{MeV}$, i.e., roughly $1 \mathrm{MeV}$ below the experiment. A similar ab initio NCCI calculation in Ref. [46] with next-to-next-to-leading order (NNLO) chiral potential results in underbounded g.s. energy.

As shown in the figure, our calculations reproduce relatively well odd-spin states. The level of agreement is comparable to, if not better than, the $a b$ initio NCCI results. The calculated isospin doublet of $1^{+}$states around $24 \mathrm{MeV}$ may represent a
TABLE II. Experimental and theoretical excitation energies of the three lowest $2^{+}$states in ${ }^{8} \mathrm{Be}$ and the corresponding $\log f t$ values. Experimental data are taken from Ref. [47].

\begin{tabular}{lccccc}
\hline \hline & \multicolumn{2}{c}{ Experiment } & & \multicolumn{2}{c}{ DFT-NCCI } \\
\cline { 2 - 3 } \cline { 6 - 6 } State & $E(\mathrm{MeV})$ & $\log f t$ & & $E(\mathrm{MeV})$ & $\log f t$ \\
\hline $2_{1}^{+} T=0$ & 3.030 & 5.36 & & 2.698 & 4.74 \\
$2_{2}^{+} T=0$ & 16.626 & 2.93 & & 11.869 & 3.54 \\
$2_{3}^{+} T=1$ & 16.922 & & & 12.812 & 4.13 \\
\hline \hline
\end{tabular}

doublet seen experimentally at $23 \mathrm{MeV}$. Spins for this doublet have not yet been assigned. Even-spin states, on the other hand, are systematically overbound. The lowest $2^{+}$and $4^{+}$states are interpreted as a members of a rotational band built atop the $0^{+}$ g.s. Their empirical excitation energy ratio, $R_{4 / 2} \equiv E_{4_{1}^{+}} / E_{2_{1}^{+}}$, equals 3.75 and thus is among the largest over the entire nuclear chart. Our model captures quite well the ratio, giving $R_{4 / 2}=$ 3.77. This means that our DFT-rooted calculation reproduces well the change of the moment of inertia along the band, but strongly overestimates its magnitude.

Too strong a quadrupole collectivity $\left(2_{1}^{+}\right.$and $4_{1}^{+}$are too low in energy) and missing correlations in the calculated g.s. are well seen in the GT transition strengths of $\left({ }^{8} \mathrm{Li}, 2_{\mathrm{g} . \mathrm{s}}^{+}\right)$to $\left({ }^{8} \mathrm{Be}, 2_{i}^{+}\right)$decays. The DFT-NCCI results and experimental data are compared in Table II. The DFT-NCCI results are calculated within $1 \mathrm{p}-1 \mathrm{~h}$ configuration space. Inclusion of $2 \mathrm{p}-2 \mathrm{~h}$ configurations has only a marginal impact on the results. The transition strength to the $2_{1}^{+}$state is clearly overestimated by our model, in contrast to the transition strength to the $2_{2}^{+}$resonance, which seems to be underestimated. One should bear in mind, however, that the empirical strength to the resonance is uncertain and can be affected by the close-lying $2_{3}^{+}, T=1$ state.

\section{Gamow-Teller sum rule: Configuration and model space dependence}

The Gamow-Teller sum rule (GTSR) is commonly considered as a convenient indicator of the completeness of a model space. Under the assumption of completeness, the GTSR reads as follows:

$$
\frac{1}{g_{\mathrm{A}}^{2}} \sum_{f}\left[B_{\mathrm{GT}}^{-}\left(I_{i}^{\pi} \rightarrow I_{f}^{\pi}\right)-B_{\mathrm{GT}}^{+}\left(I_{i}^{\pi} \rightarrow I_{f}^{\pi}\right)\right]=3(N-Z),
$$

where the sum extends over all final states $I_{f}=I_{i}+k$ with $k=0, \pm 1$. The strength is defined as

$$
B_{\mathrm{GT}}^{ \pm}\left(I_{i}^{\pi} \rightarrow I_{f}^{\pi}\right)=g_{\mathrm{A}}^{2} \frac{\left|M_{\mathrm{GT}}^{ \pm}\right|^{2}}{2 I_{i}+1},
$$

where $M_{\mathrm{GT}}^{ \pm}$stands for the reduced matrix element for the Gamow-Teller one-body operator.

In this section we shall discuss the GTSR in ${ }^{8} \mathrm{Li}$ calculated within the DFT-NCCI, with a particular emphasis on its dependence on the configuration and model spaces. The configurations (HF solutions) are numbered and labeled as in Table I. The model space, on the other hand, is spanned by 


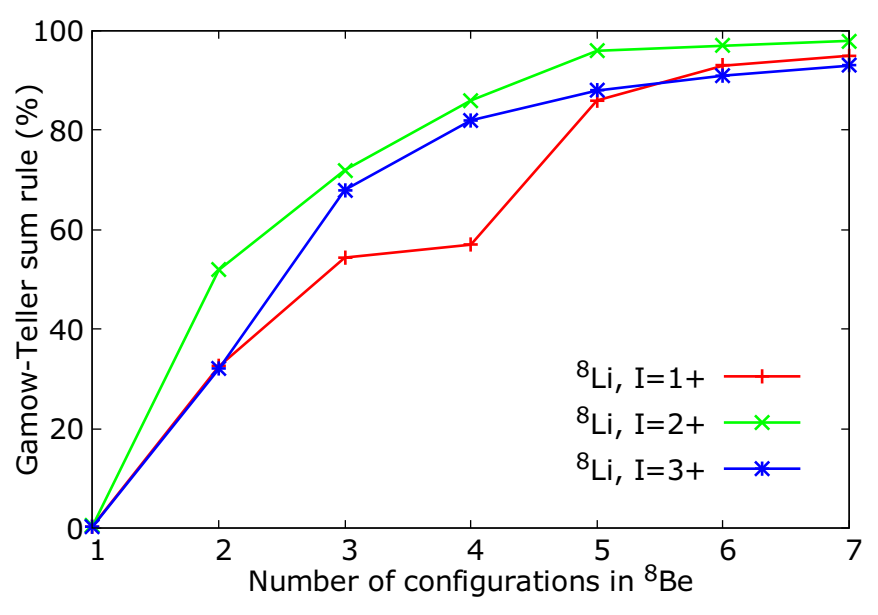

FIG. 6. GTSRs for the lowest $1_{1}^{+}, 2_{1}^{+}$, and $3_{1}^{+}$initial state in ${ }^{8} \mathrm{Li}$ against the number of configurations included in ${ }^{8} \mathrm{Be}$. The configurations are listed in Table I.

so-called natural states. These are linearly independent linear combinations of projected states, Eq. (4), having eigenvalues of the norm matrix, $n_{i}$, larger than a certain externally provided cutoff parameter $\varepsilon$.

In Fig. 6 we show the saturation of GTSRs for the ${ }^{8} \mathrm{Li} 1_{1}^{+}$, $2_{1}^{+}$, and $3_{1}^{+}$initial states versus a number of configurations used in the ${ }^{8} \mathrm{Be}$ final state. In the calculations, $B_{\mathrm{GT}}^{+}$was kept fixed at a value calculated using the entire $1 \mathrm{p}-1 \mathrm{~h}$ configuration space in ${ }^{8} \mathrm{He}$ and ${ }^{8} \mathrm{Li}$; see Table I. It is beneficial to observe that, already with five configurations in ${ }^{8} \mathrm{Be}$, the calculated GTSR reaches a level of $90 \%$. The remaining $2 \mathrm{p}-2 \mathrm{~h}$ provide circa $5 \%$ of the strength. It is interesting to note also that the unconverged 1p-1h configuration involving the $|1013 / 2\rangle$ Nilsson orbit can be effectively replaced by $2 \mathrm{p}-2 \mathrm{~h}$ excitations to this orbital.

Figure 7 shows the GTSRs in ${ }^{8} \mathrm{Li}$ and their sensitivity with respect to the cutoff parameter $\varepsilon$. The calculations are performed for $2_{1}^{+}, 1_{1}^{+}$, and $3_{1}^{+}$states in ${ }^{8} \mathrm{Li}$. In the calculations we fix the number of configurations in ${ }^{8} \mathrm{Be}$ and ${ }^{8} \mathrm{He}$, by taking

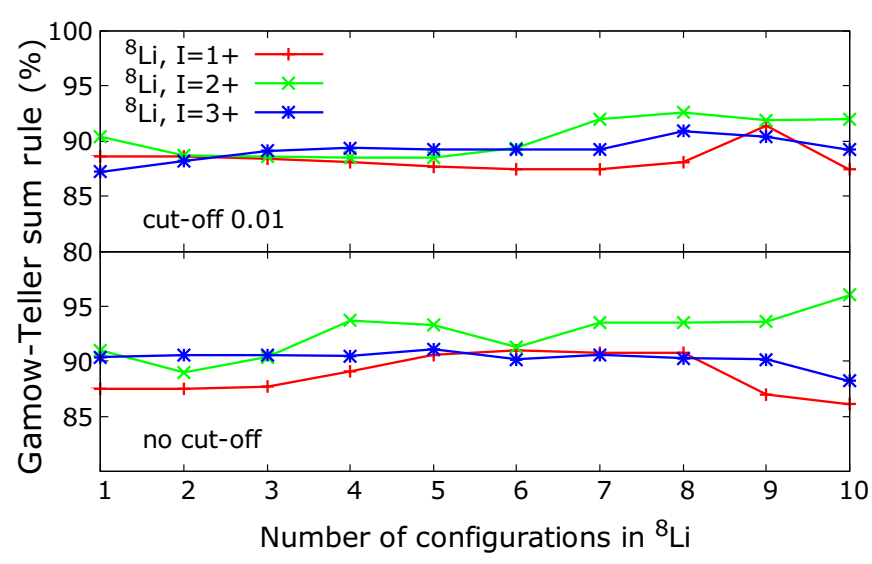

FIG. 7. GTSRs for the first $1_{1}^{+}, 2_{1}^{+}$, and $3_{1}^{+}$initial state in ${ }^{8} \mathrm{Li}$ against the number of configurations included in ${ }^{8} \mathrm{Li}$. The bottom panel shows the GTSR calculated without the cutoff. The upper panel represents the results obtained for $\varepsilon \approx 0.01$. See text for further details.

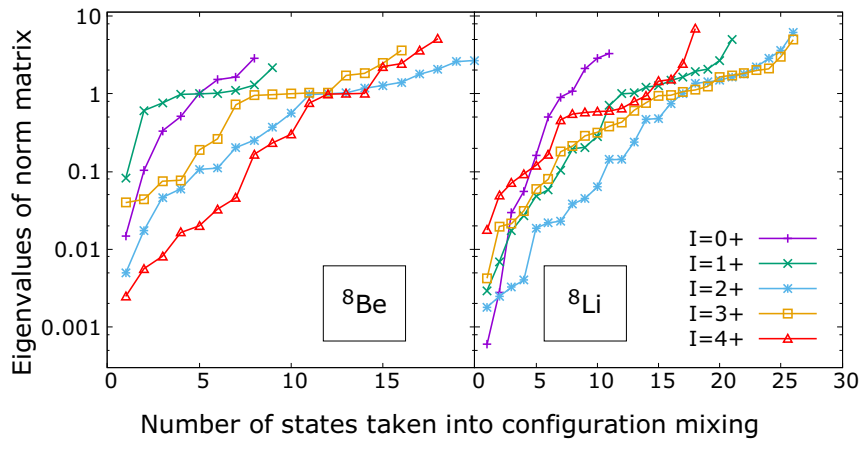

FIG. 8. Eigenvalues of the norm matrix for $0^{+}, 1^{+}, 2^{+}, 3^{+}$, and $4^{+}$states in ${ }^{8} \mathrm{Be}$ (left) and in ${ }^{8} \mathrm{Li}$ (right). The eigenvalues are plotted in ascending order.

five configurations in each nucleus, and add configurations in ${ }^{8} \mathrm{Li}$ to study the saturation of the GTSR. In the bottom panel we present the results without any cutoff. With a single g.s. configuration in ${ }^{8} \mathrm{Li}$ we reach $\sim 90 \%$ of the sum rule, irrespectively of spin. The GTSR value does not change much with increasing number of included configurations in ${ }^{8} \mathrm{Li}$. The reason comes from the fact that within our framework the lowest-lying $2_{1}^{+}, 1_{1}^{+}$, and $3_{1}^{+}$states have their origins in the g.s. configuration, which captures most of the important correlations. The calculated binding energies of these states are also relatively stable, although their stability is somewhat more sensitive to the applied cutoff parameters.

In many cases the natural states, corresponding to small eigenvalues of the norm matrix, lead to instabilities in DFTNCCI calculation. The instabilities can be controlled to some extent by applying the appropriate cutoff parameter $\varepsilon$. The choice of the cutoff parameter is, however, not unique. Typically, its value is correlated with discontinuities (or jumps) seen in the eigenvalues of the norm matrix plotted in ascending (or descending) order. In ${ }^{8} \mathrm{Li}$ (see Fig. 8) the most natural choice is $\varepsilon \approx 0.01$. This choice, as shown in Fig. 7, has almost no impact on either the GTSR or the GTSD. With increasing $\varepsilon$, more physical states are being removed, which, in turn, gives rise to large variations of the GTSR and GTSD versus number of configurations.

\section{GAMOW-TELLER STRENGTH DISTRIBUTION IN THE $s d$-MIDSHELL NUCLEUS ${ }^{24} \mathrm{Mg}$}

In this section we present the DFT-NCCI results for the Gamow-Teller strength distribution (GTSD) in ${ }^{24} \mathrm{Mg}$ following the g.s. $\beta$ decay of ${ }^{24} \mathrm{Al}\left(I_{\text {g.s. }}^{\pi}=4^{+}\right)$. For similar analysis of the GTSD in the neighboring nucleus ${ }^{20} \mathrm{Ne}$, we refer the reader to our conference publication [48].

Within the conventional spherical shell-model terminology, ${ }^{24} \mathrm{Mg}$ is an $s d$-shell nucleus having eight valence particles. Mean-field calculations, on the other hand, predict ${ }^{24} \mathrm{Mg}$ to be a well deformed system. Hence, the DFT-NCCI configuration space is built by promoting particles among the deformed s.p. Nilsson levels as shown in Fig. 9.

In order to facilitate the discussion below, let us recall that the Nilsson levels $|2201 / 2\rangle,|2113 / 2\rangle$, and $|2025 / 2\rangle$ 


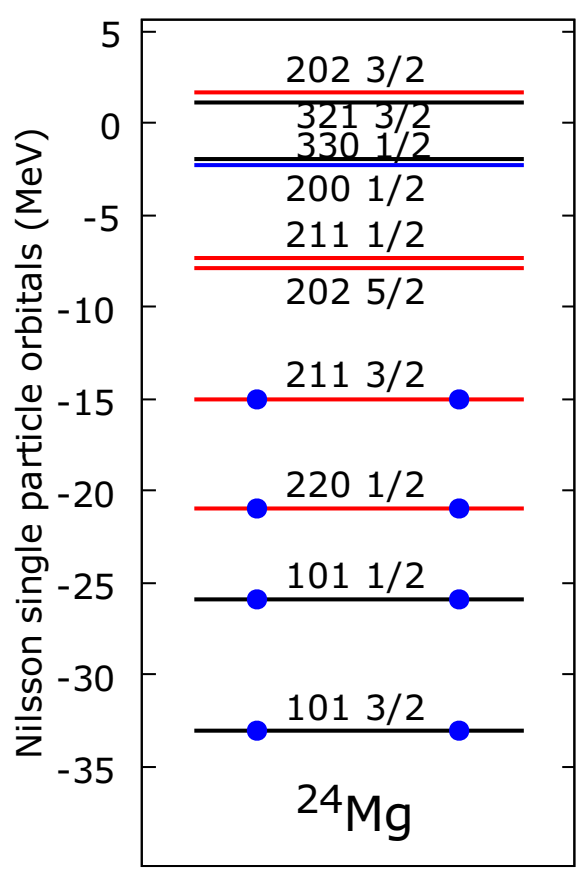

FIG. 9. Neutron mean-field s.p. levels in the g.s. of ${ }^{24} \mathrm{Mg}$. The levels are labeled using the asymptotic Nilsson quantum numbers. Dots indicate occupied levels.

originate from the spherical $d_{5 / 2}$ subshell, the level $|2001 / 2\rangle$ comes from the spherical $s_{1 / 2}$ subhell, and the levels $|2111 / 2\rangle$ and $|2023 / 2\rangle$ originate from the spherical $d_{3 / 2}$ subshell. Moreover, the levels $|2001 / 2\rangle$ and $|2111 / 2\rangle$ are predicted to mix through the quadrupole field when intrinsic deformation is $\beta_{2} \sim 0.1-0.3$; see for example the Nilsson diagram in Ref. [36].

\section{A. Configuration space}

The configuration spaces for ${ }^{24} \mathrm{Mg}$ are built by following the general rules sketched in Sec. II. We include the ground state and all possible $1 \mathrm{p}-1 \mathrm{~h}$ excitations among active $N=2$ Nilsson levels shown in Fig. 9. In self-conjugated nuclei the isospin projection allows one to reduce the space by considering $\mathrm{p}-\mathrm{h}$ excitations of a single, e.g., neutron, charge. Indeed, the proton s.p. levels are almost identically spaced and just pushed higher in energy due to the Coulomb interaction. Simple counting shows that there are 16 different $\nu \mathrm{p}-\nu \mathrm{h}$ excitations. In addition, we include in the configuration spaces two lowest $2 \mathrm{p}-2 \mathrm{~h}$ configurations. These configurations are added in order to test stability of the GTSD with respect to higher order excitations. All HF states included in the configuration space of ${ }^{24} \mathrm{Mg}$ are listed in Table III. They are prolate deformed axially symmetric configurations.

A systematic study of the GT matrix elements (GTMEs) in $T=1 / 2$ mirror nuclei [8] allowed us to conclude that these g.s. to g.s. $I^{\pi} \rightarrow I^{\pi}$ matrix elements are fairly insensitive on the configuration mixing. A similar property appears to hold here, as demonstrated in Fig. 10. The figure shows calculated $4^{+} \rightarrow 4^{+}$GTMEs between the g.s. of ${ }^{24} \mathrm{Al}$ and the $4^{+}$states in ${ }^{24} \mathrm{Mg}$ calculated by using the DFT-NCCI
TABLE III. The list of configurations in ${ }^{24} \mathrm{Mg}$, labeled by the index $i$ and asymptotic Nilsson quantum numbers of excited p-h states. Listed are also the $\mathrm{HF}$ binding energy $E_{\mathrm{HF}}$ in $\mathrm{MeV}$, excitation energy $\Delta E$ in $\mathrm{MeV}$, quadrupole deformation parameters $\beta_{2}$, and the total alignment $K$ together with its orientation in the intrinsic frame.

\begin{tabular}{|c|c|c|c|c|c|}
\hline $\boldsymbol{l}$ & $\left.{ }^{24} \mathrm{Mg} ; i\right\rangle$ & $E_{\mathrm{HF}}$ & $\Delta E$ & $\beta_{2}$ & $K$ \\
\hline 1 & g.s. & -194.33 & 0 & 0.42 & 0 \\
\hline 2 & $|\nu 2113 / 2-\rangle^{-1} \otimes|v 2025 / 2-\rangle^{1}$ & -187.92 & 6.41 & 0.34 & \\
\hline 3 & $|\nu 2113 / 2+\rangle^{-1} \otimes|\nu 2025 / 2-\rangle^{1}$ & -187.25 & 7.08 & 0.34 & $4_{z}$ \\
\hline 4 & $|v 2113 / 2+\rangle^{-1} \otimes|v 2111 / 2-\rangle^{1}$ & -187.46 & 6.87 & 0.43 & $2 z$ \\
\hline 5 & $|\nu 2113 / 2-\rangle^{-1} \otimes|\nu 2111 / 2-\rangle^{1}$ & -184.89 & 9.44 & 0.40 & \\
\hline 6 & $|v 2201 / 2-\rangle^{-1} \otimes|v 2025 / 2-\rangle^{1}$ & -183.34 & 10.99 & 0.24 & 27 \\
\hline 7 & $|v 2201 / 2+\rangle^{-1} \otimes \mid \nu$ & -183.27 & 11.06 & 0.23 & $3_{Z}$ \\
\hline 8 & $|v 2113 / 2+\rangle^{-1} \otimes \mid v 2$ & -181.79 & 12.54 & 0.36 & \\
\hline 9 & $|\nu 2113 / 2+\rangle^{-1} \otimes|\nu 2001 / 2-\rangle^{1}$ & -181.50 & 12.83 & 0.34 & \\
\hline 10 & $|v 2201 / 2+\rangle^{-1} \otimes \mid v$ & -181.99 & 12.34 & 0.35 & 1 工 \\
\hline 11 & $|v 2201 / 2-\rangle^{-1} \otimes|v 2111 / 2-\rangle^{1}$ & -180.78 & 13.55 & 0.33 & $0_{z}$ \\
\hline 12 & $|\nu 2113 / 2-\rangle^{-1} \otimes|\nu 2023 / 2+\rangle^{1}$ & -178.83 & 15.50 & 0.34 & \\
\hline 13 & $|\nu 2113 / 2+\rangle^{-1} \otimes|\nu 2023 / 2+\rangle^{1}$ & -177.16 & 17.17 & 0.33 & \\
\hline 14 & $|\nu 2201 / 2-\rangle^{-1} \otimes|\nu 2001 / 2-\rangle^{1}$ & -177.04 & 17.29 & 0.27 & $0_{2}$ \\
\hline 15 & $|v 2201 / 2+\rangle^{-1} \otimes|v 2001 / 2-\rangle^{1}$ & -176.94 & 17.39 & 0.25 & \\
\hline 16 & $|\nu 2201 / 2-\rangle^{-1} \otimes|\nu 2023 / 2+\rangle^{1}$ & -174.00 & 20.33 & 0.25 & \\
\hline 17 & $|v 2113 / 2+\rangle^{-1} \otimes \mid v 2$ & -173.47 & 20.86 & 0.24 & \\
\hline 18 & $|\pi 2113 / 2-\rangle^{-1} \otimes|\pi 2025 / 2-\rangle^{1}$ & -188.00 & 6.33 & 0.34 & \\
\hline 19 & $|\nu 2113 / 2-\rangle^{-1} \otimes|\nu 2025 / 2-\rangle^{1}$ & -184.29 & 10.04 & 0.10 & \\
\hline & $|\pi 2113 / 2-\rangle^{-1} \otimes|\pi 2025 / 2-\rangle^{1}$ & & & & \\
\hline 20 & $|v 2113 / 2\rangle^{-2} \otimes|\nu 2025 / 2\rangle^{2}$ & -183.13 & 11.20 & 0.26 & $0_{z}$ \\
\hline
\end{tabular}

model with 17 configurations involving the g.s. and all $1 \mathrm{p}-1 \mathrm{~h}$ excitations. Each of the panels differs by the treatment of the g.s. DFT-NCCI wave function of the parent nucleus. We start with the wave function projected from the so-called aligned SR g.s. configuration (a) and enrich it by admixing first the anti-aligned g.s. (b) and, eventually, the lowest $1 \mathrm{p}-1 \mathrm{~h}$ excitation (c). As is clearly visible, the calculated GTMEs are almost insensitive to the wave function in the parent nucleus. Hence, in all calculations shown below, the correlated g.s. DFT-NCCI wave function in ${ }^{24} \mathrm{Al}$ includes three SR Slater determinants: the aligned g.s., the anti-aligned g.s., and the lowest $1 \mathrm{p}-1 \mathrm{~h}$ excitation.

\section{B. Gamow-Teller strength distribution}

In order to pin down a specific role played by different Nilsson levels, we studied diagonal $I^{\pi} \rightarrow I^{\pi}$ GTMEs as a function of the configuration space size in the daughter nucleus. The results are illustrated in Fig. 11. The bottom panel (a) shows the GTME distribution calculated using HF configurations involving the g.s. and all $1 \mathrm{p}-1 \mathrm{~h}$ excitations among the Nilsson levels originating from the spherical $d_{5 / 2}$ subshell. Panel (b) contains additionally all $1 \mathrm{p}-1 \mathrm{~h}$ configurations involving the $|2111 / 2\rangle$ Nilsson state originating from the spherical $d_{3 / 2}$ subshell. This level plays a critical role in shaping up the GTSD in ${ }^{24} \mathrm{Mg}$ around the excitation energy of $\sim 8 \mathrm{MeV}$. This example 


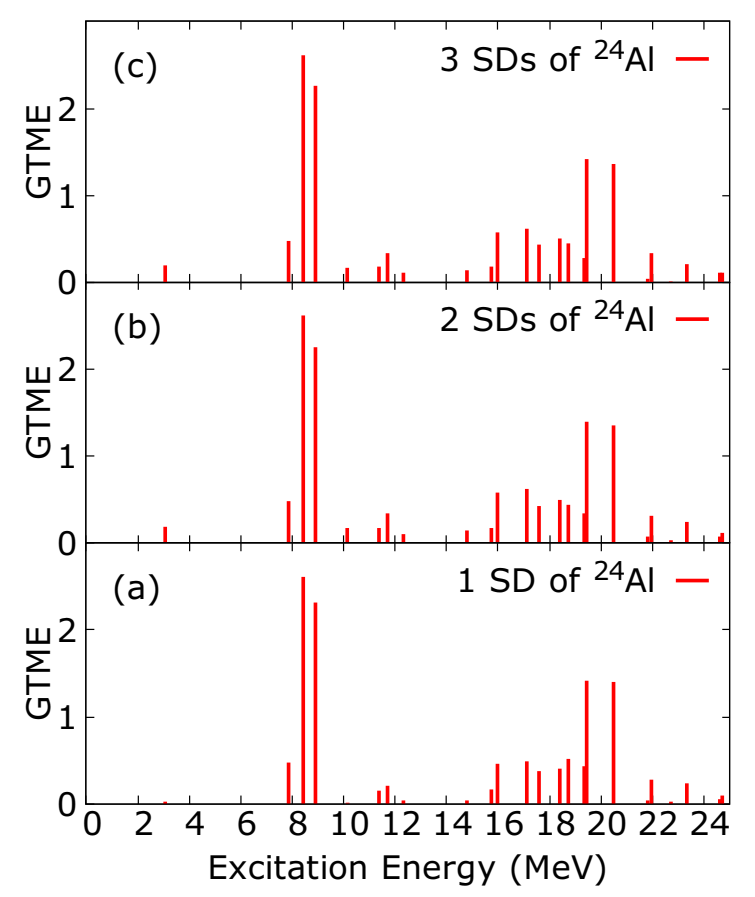

FIG. 10. GTME stability analysis against configuration mixing in the parent nucleus. The figure shows GTMEs for $\left|{ }^{24} \mathrm{Al} ; 4_{\mathrm{g.s} .}^{+}\right\rangle \rightarrow$ $\left.\left.\right|^{24} \mathrm{Mg} ; 4^{+}\right\rangle$decay. The results were obtained by using 17 configurations in the daughter nucleus. The number of Slater determinants (SDs) used to correlate the parent nucleus changes from 1 (bottom) to 3 (top).

shows how sensitive the GTSD is with respect to the position of s.p. levels. Panel (c) shows the GTMEs calculated using all HF configurations having excitation energies $\Delta E_{\mathrm{HF}}$ below the experimental $Q_{\beta}$ value, i.e., $\Delta E_{\mathrm{HF}} \leqslant 14.5 \mathrm{MeV}$. Finally, (d) shows the distribution calculated using all available $\mathrm{p}-\mathrm{h}$ configurations. The configurations included in panels (c) and (d) influence predominantly the high-energy part of GTME distribution, above the experimental $Q_{\beta}$ window.

The result from the DFT-NCCI calculation, including transitions from the $4^{+}$g.s. of ${ }^{24} \mathrm{Al}$ to all $3^{+}, 4^{+}$, and $5^{+}$ states in ${ }^{24} \mathrm{Mg}$, is shown in Fig. 12. The calculated GTSD is compared to the USDb shell-model calculation and experiment [49]. Both the shell model and DFT-NCCI results are in a perfect agreement with experiment concerning position of a centroid describing the $4^{+} \rightarrow 4^{+}$transition, but the theoretical peaks are roughly two times higher compared to experiment. Moreover, in the DFT-NCCI calculations the first resonant peak, called hereafter the first GT resonance (GTR1), splits into two close-lying peaks. The second GT resonance (GTR2) seen in the DFT-NCCI calculation at high excitations energies is well above the experimental $Q_{\beta}$ energy.

In order to reveal the nature of resonant transitions, their wave functions has been decomposed in terms of HF configurations, shown in Fig. 13. The $4^{+}$g.s. of ${ }^{24} \mathrm{Al}$ is dominated by the aligned $\mathrm{HF}$ configuration having an unpaired proton on the $|2025 / 2\rangle$ s.p. level. This level has large GT s.p. matrix elements with the $|2025 / 2\rangle$ level and its spin-orbit partner, $|2023 / 2\rangle$, in ${ }^{24} \mathrm{Mg}$. Hence, the GTR1 is due to transition to

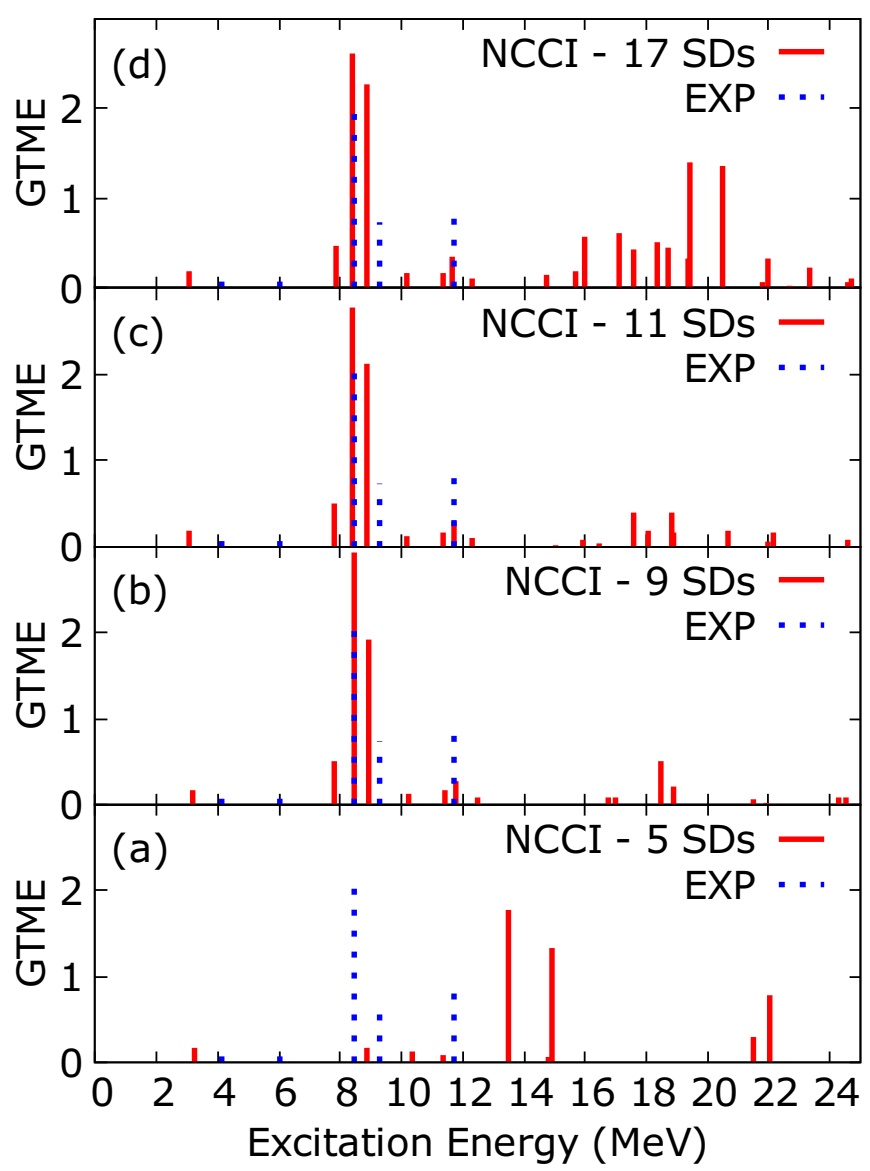

FIG. 11. GTMEs between the $4^{+}$g.s. of ${ }^{24} \mathrm{Al}$ and the $4^{+}$states in ${ }^{24} \mathrm{Mg}$ for various dimensions of the used configuration space in ${ }^{24} \mathrm{Mg}$. See text for details.

the aligned $\mathrm{p}$-h excitation involving the neutron particle in $|2025 / 2\rangle$. Its structure, however, is strongly affected by the aligned p-h excitation involving the neutron particle in the $|2111 / 2\rangle$ orbit due to proximity of the $|2025 / 2\rangle$ and $|2111 / 2\rangle$

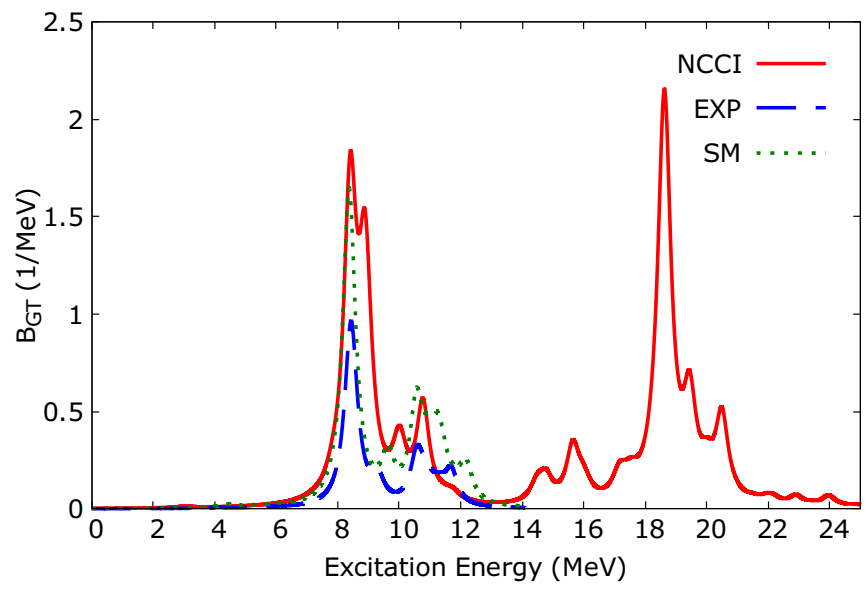

FIG. 12. Complete GTSD in ${ }^{24} \mathrm{Mg}$ following $\beta$ decay of the $4^{+}$ g.s. in ${ }^{24} \mathrm{Al}$. The GTSD includes matrix elements for $3^{+}, 4^{+}$, and $5^{+}$ states in ${ }^{24} \mathrm{Mg}$. The DFT-NCCI result is compared to experimental data and the shell-model calculations of Ref. [49]. Curves are smoothed with a Lorentzian function with a half-width of $\Gamma=0.5 \mathrm{MeV}$. 


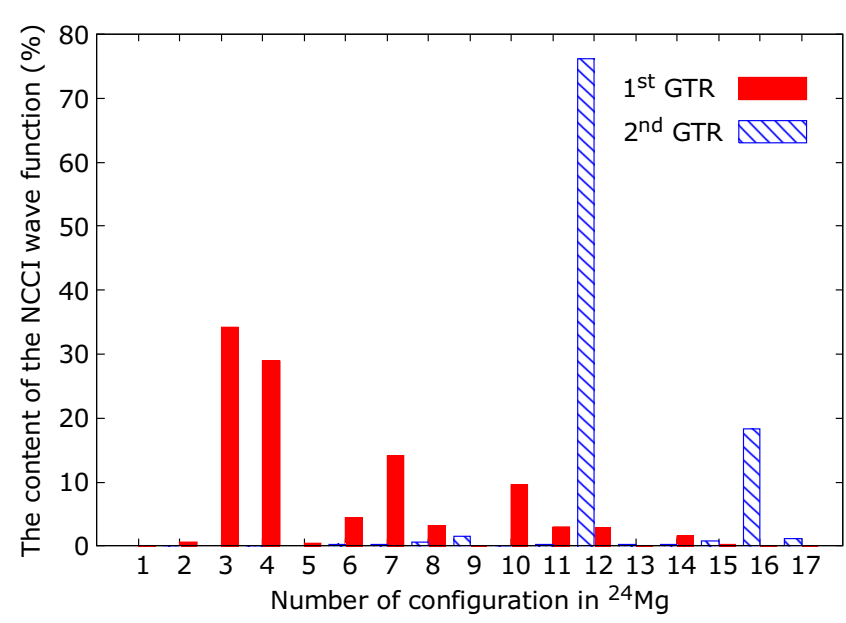

FIG. 13. The content of the wave function of first $4^{+}$GamowTeller resonance (red) and of the second GTR peak in terms of HF configurations listed in Table III.

levels in the potential well; see Fig. 9. The near-degeneracy causes mixing between the states projected from these HF configurations, since the $K$ quantum number is not conserved.

By slightly increasing the spacing between the $|2025 / 2\rangle$ and $\mid 211$ 1/2 $\rangle$ s.p. levels, the mixing becomes reduced, which increases purity of the GTR1 wave function and further improves agreement with the data. The spacing can be increased, for example, by slight increase of the spin-orbit strength. The result of such a test study is shown in Fig. 14. The figure shows a series of DFT-NCCI calculations using the $\mathrm{SV}_{\mathrm{T}}$ Skyrme force with the spin-orbit strength increased by $10 \%, 20 \%, 30 \%$, and $40 \%$ with respect to the original value. The configuration space in ${ }^{24} \mathrm{Mg}$ used in this test study was constrained to the SR g.s. and two aligned p-h excitations to $|2025 / 2\rangle$ and $|2111 / 2\rangle$ levels. The calculation shows that the centroid of the main peak and its height weakly depend on the spin-orbit strength, at variance with the secondary peak associated with the $\mid 211$ 1/2〉 Nilsson level. Indeed, with increasing spin-orbit strength the secondary peak moves toward higher energies and its magnitude decreases. The study suggest that the optimal spin-orbit strength should be around 25\% larger than the

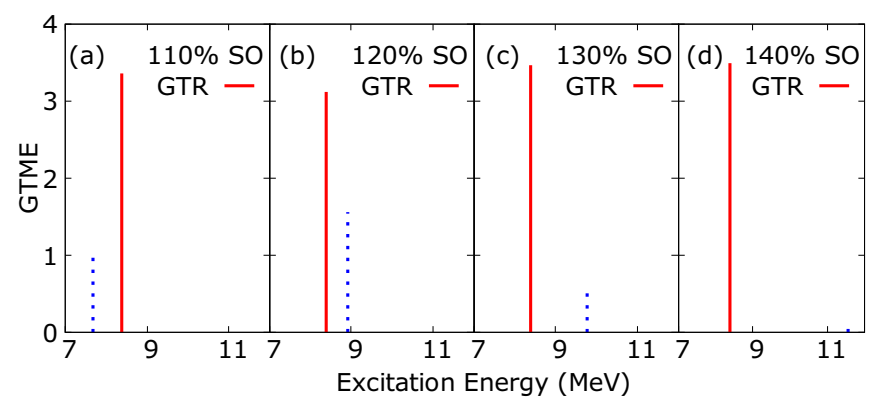

FIG. 14. GTMEs connecting the $4^{+}$g.s. in ${ }^{24} \mathrm{Al}$ with the $|2025 / 2\rangle$ resonant peak (solid line) and the $|2111 / 2\rangle$ secondary peak (dotted line). The calculations were done using the $\mathrm{SV}_{\mathrm{T}}$ parametrization with spin-orbit strength increased by $10 \%$ (a), $20 \%$ (b), $30 \%$ (c), and $40 \%$ (d) with respect to the original value. See text for further details. original value. In contrast to the GTR1, the structure of GTR2 is predicted to be very pure, with $80 \%$ of its wave function content coming from the aligned $1 \mathrm{p}-1 \mathrm{~h}$ excitation involving the neutron particle in the $|2023 / 2\rangle$ Nilsson level.

\section{SUPERALLOWED GAMOW-TELLER $\beta$ DECAY OF ${ }^{100} \mathrm{Sn}$}

In this section we have computed the superallowed GamowTeller $\beta$ decay of the heaviest $N=Z$ nucleus ${ }^{100} \mathrm{Sn}$ and the low-spin structure, $I \leqslant 8$, in the daughter nucleus ${ }^{100} \mathrm{In}$. The transition, which proceeds from the $0^{+}$g.s. of ${ }^{100} \mathrm{Sn}$ to the first $1_{1}^{+}$state in ${ }^{100} \mathrm{In}$, is the fastest GT decay observed so far; see Ref. [50]. The GTME is well reproduced by the dedicated large-scale shell-model (LSSM) calculations under the assumption that the axial coupling constant is quenched by $40 \%[50]$.

The aim is to test the universality of the DFT-NCCI approach which, at least in principle, can be applied to calculate both the nuclear spectra and transition rates in any atomic nucleus, irrespectively of its mass and particle-number parity. Hence, in the calculation we have used exactly the same formalism as in the preceding sections. The HF configurations were calculated by using the $\mathrm{SV}_{\mathrm{SO}}$ variant of the Skyrme $\mathrm{SV}_{\mathrm{T}}$ force within the space consisting of 12 spherical harmonic oscillator shells.

The $\mathrm{SV}_{\text {SO }}$ has a $20 \%$ stronger spin-orbit interaction strength compared to $\mathrm{SV}_{\mathrm{T}}$ [8]. As discussed in Ref. [8], the use of the $\mathrm{SV}_{\text {so }}$ variant considerably improves calculated masses in $N \sim$ $Z$ nuclei as compared to the DFT-NCCI calculations based on the $\mathrm{SV}_{\mathrm{T}}$ force.

In the case of the doubly-magic ${ }^{100} \mathrm{Sn}$, we considered only a single mean field configuration representing its g.s. The calculated binding energy $827.7 \mathrm{MeV}$ of ${ }^{100} \mathrm{Sn}$ is in fair agreement with the experimental value $825.3 \pm 0.3 \mathrm{MeV}$, overestimating it by $0.3 \%$.

The structure of ${ }^{100}$ In was computed by using nine axially deformed mean field configurations. Counting with respect to the ${ }^{100} \mathrm{Sn}$ core, eight of them correspond to p-h configurations, with the neutron particle occupying different s.p. states originating from the $d_{5 / 2}$ and $g_{7 / 2}$ spherical subshells and the proton hole being in the s.p. orbital originating from the $g_{9 / 2}$ spherical sub-shell. In spherical language these are $v d_{5 / 2} \otimes \pi g_{9 / 2}^{-1}$ and $v f_{5 / 2} \otimes \pi g_{9 / 2}^{-1}$ configurations. The ninth configuration, involving the lowest $\pi \mathrm{p}-\pi \mathrm{h}$ excitation through the $Z=50$ shell gap, was added to test stability against the cross-shell excitations. The calculation shows that it does not affect either the low-lying spectrum or the GTME.

The calculated spectrum, which includes the first $1^{+} \leqslant$ $I^{\pi} \leqslant 8^{+}$states in ${ }^{100} \mathrm{In}$, is depicted in Fig. 15 and compared to the LSSM results for the first $1^{+} \leqslant I^{\pi} \leqslant 6^{+}$, taken from Ref. [50]. We refrain from showing the experimental spectrum since neither the spins nor the excitation energies are firmly assigned [50]. Theoretical spectra were normalized to the g.s. energy, which is predicted to have $I=6^{+}$by both the models. The predicted DFT-NCCI binding energy for this state is in perfect agreement with the experimental binding energy of ${ }^{100} \mathrm{In}$, underestimating it only by $9 \mathrm{keV}$. Concerning excited states, the DFT-NCCI model predicts the following values: $0.618 \mathrm{MeV}\left(5_{1}^{+}\right), 0.637 \mathrm{MeV}\left(7_{1}^{+}\right), 0.927 \mathrm{MeV}\left(8_{1}^{+}\right)$, 


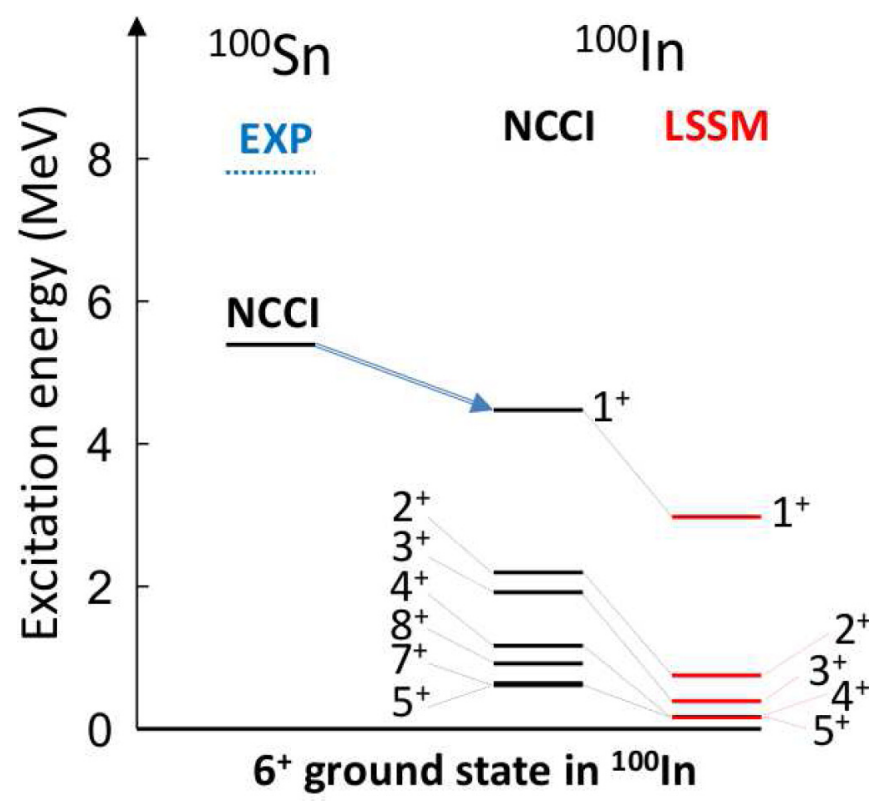

FIG. 15. Low-lying states in ${ }^{100}$ In calculated by using the DFTNCCI (middle) and LSSM (right). The left part shows the experimental (dotted line) and DFT-NCCI (solid line) binding energies in ${ }^{100} \mathrm{Sn}$ relative to the ground state energy in ${ }^{100} \mathrm{In}$. See text for details.

$1.176 \mathrm{MeV}\left(4_{1}^{+}\right), 1.912 \mathrm{MeV}\left(3_{1}^{+}\right), 2.194 \mathrm{MeV}\left(2_{1}^{+}\right)$, and $4.475 \mathrm{MeV}\left(1_{1}^{+}\right)$. The excitation energy of $8^{+}$state may be somewhat uncertain due to a too small number of knots used in the integration over the angles in the angular-momentum projection procedure. The level ordering agrees relatively well with the LSSM calculations but the excitation energies are systematically larger. Note, that the DFT-NCCI model predicts the low-lying doublet composed of $5_{1}^{+}$and $7_{1}^{+}$states at variance to the LSSM, which predicts near-degeneracy of the first $5_{1}^{+}$ and $4_{1}^{+}$states.

The calculated $B_{\mathrm{GT}}^{(\mathrm{NCCl})} \approx 10.2$ after using the effective axial-vector strength $g_{\mathrm{A}}^{\text {(eff) }}=q g_{\mathrm{A}}$, quenched by $40 \%$ [50] with respect to the free-neutron value of $g_{\mathrm{A}}=-1.2701$. The quenching factor $q=0.6$ is typical for the $A \approx 100$ mass region [51]. The quenched $B_{\mathrm{GT}}^{(\mathrm{NCCl})}$ agrees well with the experimental value $B_{\mathrm{GT}}^{(\mathrm{EXP})}=9.1_{-3.0}^{+2.6}$.

\section{CONCLUSIONS}

In the present work we have presented pioneering calculations of the Gamow-Teller transitions by using the no-core configuration-interaction approach based on multireference density functional theory, treating properly the isospin and rotational symmetries. The DFT-NCCI formalism was applied to compute the GTSD in the $p$-shell ${ }^{8} \mathrm{Li}$ and ${ }^{8} \mathrm{Be}$ nuclei.
Although the model lacks the coupling to continuum essential to describe broad resonances and in turn $\beta$-decay properties, we have shown that it can provide an input to theories exploring open-channel physics, such as the $R$ matrix. Shell-model calculation applied to such an approach supported experimental-data analysis. It may be of particular interest to follow the path with an entirely different DFT-rooted theory. Moreover, we have demonstrated that the model is capable of capturing the GTSD satisfactorily well using a relatively small configuration space in the $s d$-shell ${ }^{24} \mathrm{Mg}$ as well. It was also shown that the model allows for interpretation of the GTSD peaks in terms of specific Nilsson orbits of a deformed mean field, i.e., in a way that is complementary to the traditional nuclear shell-model calculations.

The DFT-NCCI model can be, at least in principle, applied to calculate both the nuclear spectra and transition rates in atomic nuclei irrespectively of their mass and particle-number parity. In order to demonstrate its flexibility, the model was also applied to compute the superallowed GT $\beta$ decay in ${ }^{100} \mathrm{Sn}$ and the low-spin spectrum in ${ }^{100} \mathrm{In}$. It is shown that, after applying the standard quenching factor of $q \approx 0.6$, the calculated matrix element agrees well with the experimental value. The low-spin spectrum agrees quite well with the large-space shell-model calculation of Hinke [50]. Finally, let us stress that all the results presented above were obtained without any readjustment of the model parameters to experimental data.

In conclusion, we have demonstrated that the DFT-NCCI formalism can be successfully used to study nuclear $\beta$ decay in a diverse set of nuclei, thus offering a complementary method to ab initio and shell-model approaches. This study paves a way for more systematic studies of nuclear $\beta$-decay rates, for exploring forbidden $\beta$ decays, and for tackling the double- $\beta$-decay process within the DFT-NCCI framework. With forbidden $\beta$ decays, the spectrum-shape method may offer valuable hints for the $g_{\mathrm{A}}$ quenching puzzle [52]. Although the correspondence to experimental results was generally found to be rather good, the underlying effective interaction used to construct the EDF has its limitations. A work towards developing novel EDFs applicable for beyond-mean-field calculations is under way.

\section{ACKNOWLEDGMENTS}

This work was supported by the Polish National Science Center (NCN) under Contracts No. 2014/15/N/ST2/03454 and No. 2017/24/T/ST2/00160, by the Academy of Finland under the Centre of Excellence Programme 2012-2017 (Nuclear and Accelerator Based Physics Programme at JYFL), and by the FIDIPRO program. We acknowledge the CIS-IT National Centre for Nuclear Research (NCBJ), Poland and CSC-IT Center for Science Ltd., Finland for allocation of computational resources.
[1] M. Bender, P.-H. Heenen, and P.-G. Reinhard, Rev. Mod. Phys. 75, 121 (2003).
[2] J. Erler, N. Birge, M. Kortelainen, W. Nazarewicz, E. Olsen, A. M. Perhac, and M. Stoitsov, Nature (London) 486, 509 (2012). 
[3] E. Caurier, G. Martínez-Pinedo, F. Nowacki, A. Poves, and A. P. Zuker, Rev. Mod. Phys. 77, 427 (2005).

[4] B. Bally, B. Avez, M. Bender, and P.-H. Heenen, Phys. Rev. Lett. 113, 162501 (2014).

[5] W. Satuła, J. Dobaczewski, M. Konieczka, and W. Nazarewicz, Acta Phys. Pol. B 45, 167 (2014).

[6] W. Satuła, J. Dobaczewski, and M. Konieczka, JPS Conf. Proc. 6, 020015 (2015).

[7] W. Satuła, P. Bączyk, J. Dobaczewski, and M. Konieczka, Phys. Rev. C 94, 024306 (2016).

[8] M. Konieczka, P. Bączyk, and W. Satuła, Phys. Rev. C 93, 042501 (2016).

[9] J. L. Egido, M. Borrajo, and T. R. Rodríguez, Phys. Rev. Lett. 116, 052502 (2016).

[10] T. R. Rodríguez, A. Poves, and F. Nowacki, Phys. Rev. C 93, 054316 (2016).

[11] P. W. Zhao, P. Ring, and J. Meng, Phys. Rev. C 94, 041301 (2016).

[12] M. Borrajo and J. L. Egido, Phys. Lett. B 764, 328 (2017).

[13] J. Menéndez, D. Gazit, and A. Schwenk, Phys. Rev. Lett. 107, 062501 (2011).

[14] A. Ekström, G. R. Jansen, K. A. Wendt, G. Hagen, T. Papenbrock, S. Bacca, B. Carlsson, and D. Gazit, Phys. Rev. Lett. 113, 262504 (2014).

[15] A. Baroni, L. Girlanda, A. Kievsky, L. E. Marcucci, R. Schiavilla, and M. Viviani, Phys. Rev. C 94, 024003 (2016).

[16] H. De-Leon, L. Platter, and D. Gazit, arXiv:1611.10004.

[17] P. Klos, A. Carbone, K. Hebeler, J. Menéndez, and A. Schwenk, Eur. Phys. J. A 53, 168 (2017).

[18] J. T. Suhonen, Frontiers Phys. 5, 55 (2017).

[19] B. Brown, Prog. Part. Nucl. Phys. 47, 517 (2001).

[20] V. Kumar and P. C. Srivastava, Eur. Phys. J. A 52, 181 (2016).

[21] H. Nakata, T. Tachibana, and M. Yamada, Nucl. Phys. A 625, 521 (1997).

[22] P. Sarriguren, E. M. de Guerra, A. Escuderos, and A. Carrizo, Nucl. Phys. A 635, 55 (1998).

[23] J. Engel, M. Bender, J. Dobaczewski, W. Nazarewicz, and R. Surman, Phys. Rev. C 60, 014302 (1999).

[24] P. Möller, B. Pfeiffer, and K.-L. Kratz, Phys. Rev. C 67, 055802 (2003).

[25] N. Paar, T. Nikšić, D. Vretenar, and P. Ring, Phys. Rev. C 69, 054303 (2004).

[26] M. T. Mustonen and J. Engel, Phys. Rev. C 93, 014304 (2016).

[27] A. Petrovici, K. Schmid, and A. Faessler, Prog. Part. Nucl. Phys. 66, 287 (2011).
[28] J. Dobaczewski, W. Satuła, B. Carlsson, J. Engel, P. Olbratowski, P. Powałowski, M. Sadziak, J. Sarich, N. Schunck, A. Staszczak, M. Stoitsov, M. Zalewski, and H. Zduńczuk, Comput. Phys. Commun. 180, 2361 (2009).

[29] N. Schunck, J. Dobaczewski, W. Satuła, P. Bączyk, J. Dudek, Y. Gao, M. Konieczka, K. Sato, Y. Shi, X. Wang, and T. Werner, Comput. Phys. Commun. 216, 145 (2017).

[30] M. Bender, T. Duguet, and D. Lacroix, Phys. Rev. C 79, 044319 (2009).

[31] W. Satuła and J. Dobaczewski, Phys. Rev. C 90, 054303 (2014).

[32] M. Beiner, H. Flocard, N. Van Giai, and P. Quentin, Nucl. Phys. A 238, 29 (1975).

[33] J. Sadoudi, M. Bender, K. Bennaceur, D. Davesne, R. Jodon, and T. Duguet, Phys. Scr., T 154, 014013 (2013).

[34] K. Bennaceur, A. Idini, J. Dobaczewski, P. Dobaczewski, M. Kortelainen, and F. Raimondi, J. Phys. G: Nucl. Part. Phys. 44, 045106 (2017).

[35] W. Satuła, J. Dobaczewski, W. Nazarewicz, and T. R. Werner, Phys. Rev. C 86, 054316 (2012).

[36] P. Ring and P. Schuck, The Nuclear Many-Body Problem (Springer, Berlin, 1980).

[37] B. Blank and M. Borge, Prog. Part. Nucl. Phys. 60, 403 (2008).

[38] K. Riisager, Nucl. Phys. A 925, 298 (2014).

[39] F. Barker and E. Warburton, Nucl. Phys. A 487, 269 (1988).

[40] D. Tilley, J. Kelley, J. Godwin, D. Millener, J. Purcell, C. Sheu, and H. Weller, Nucl. Phys. A 745, 155 (2004).

[41] N. Kumar, Nucl. Phys. A 225, 221 (1974).

[42] M. Borge, L. Johannsen, B. Jonson, T. Nilsson, G. Nyman, K. Riisager, O. Tengblad, and K. Rolander, Nucl. Phys. A 560, 664 (1993).

[43] F. Barker, Nucl. Phys. A 609, 38 (1996).

[44] P. Maris, J. P. Vary, P. Navrátil, W. E. Ormand, H. Nam, and D. J. Dean, Phys. Rev. Lett. 106, 202502 (2011).

[45] P. Maris, M. A. Caprio, and J. P. Vary, Phys. Rev. C 91, 014310 (2015).

[46] M. A. Caprio, P. Maris, J. P. Vary, and R. Smith, Int. J. Mod. Phys. E 24, 1541002 (2015).

[47] F. Ajzenberg-Selove, Nucl. Phys. A 413, 1 (1984).

[48] M. Konieczka and W. Satuła, Acta Phys. Pol. B 48, 293 (2017).

[49] B. A. Brown and B. H. Wildenthal, At. Data Nucl. Data Tables 33, 347 (1985).

[50] C. B. Hinke et al., Nature (London) 486, 341 (2012).

[51] P. Pirinen and J. Suhonen, Phys. Rev. C 91, 054309 (2015).

[52] M. Haaranen, P. C. Srivastava, and J. Suhonen, Phys. Rev. C 93, 034308 (2016). 